\title{
The histopathology of fatal untreated human respiratory syncytial virus infection
}

\author{
Joyce E Johnson ${ }^{1}$, Ricardo A Gonzales ${ }^{2}$, Sandy J Olson ${ }^{1}$, Peter F Wright ${ }^{1,2,3}$ and \\ Barney S Graham ${ }^{4}$
}

${ }^{1}$ Department of Pathology, Vanderbilt University School of Medicine, Nashville, TN, USA; ${ }^{2}$ Department of Pediatrics, Vanderbilt University School of Medicine, Nashville, TN, USA; ${ }^{3}$ Department of Microbiology $\mathcal{\sigma}$ Immunology, Vanderbilt University School of Medicine, Nashville, TN, USA and ${ }^{4}$ Vaccine Research Center, National Institute of Allergy and Infectious Diseases, National Institutes of Health, Bethesda, MD, USA

\begin{abstract}
The pathology of respiratory syncytial virus (RSV) infection was evaluated 1 day after an outpatient diagnosis of RSV in a child who died in a motor vehicle accident. We then identified 11 children with bronchiolitis from the Vanderbilt University autopsy log between 1925 and 1959 who met criteria for possible RSV infection in the preintensivist era. Their tissue was re-embedded and evaluated by routine hematoxylin and eosin and PAS staining and immunostaining with RSV-specific antibodies. Tissue from three cases was immunostain-positive for RSV antigen and was examined in detail. Small bronchiole epithelium was circumferentially infected, but basal cells were spared. Both type 1 and 2 alveolar pneumocytes were also infected. Although, not possible for archival cases, tissue from the index case was evaluated by immunostaining with antibodies to define the cellular components of the inflammatory response. Inflammatory infiltrates were centered on bronchial and pulmonary arterioles and consisted of primarily CD69+ monocytes, CD3 + double-negative T cells, CD8 + T cells, and neutrophils. The neutrophil distribution was predominantly between arterioles and airways, while the mononuclear cell distribution was in both airways and lung parenchyma. Most inflammatory cells were concentrated submuscular to the airway, but many cells traversed the smooth muscle into the airway epithelium and lumen. Airway obstruction was a prominent feature in all cases attributed to epithelial and inflammatory cell debris mixed with fibrin, mucus, and edema, and compounded by compression from hyperplastic lymphoid follicles. These findings inform our understanding of RSV pathogenesis and may facilitate the development of new approaches for prevention and treatment.
\end{abstract}

Modern Pathology (2007) 20, 108-119. doi:10.1038/modpathol.3800725; published online 24 November 2006

Keywords: bronchiolitis; pathogenesis; asthma; immune response

The microscopic anatomy of natural respiratory syncytial virus (RSV) infection is infrequently documented $^{1-7}$ in large part because patients with acute disease are rarely biopsied. Modern day autopsy material typically reflects the features of weeks-old infection, altered by supportive therapies (positive-pressure ventilation, oxygen toxicity) and, often, lung injury from bacterial superinfection, in addition to the late effects of viral infection and host immune response. Autopsy archives before the era of mechanical ventilation and antimicrobial therapy represent a potential source of tissue from patients with fatal acute untreated RSV infection. Using an

Correspondence: Dr BS Graham, MD, PhD, Vaccine Research Center, National Institute of Allergy and Infectious Diseases, National Institutes of Health, Building 40, Room 2502, 40 Convent Drive, MSC-3017, Bethesda, MD 20892-3017, USA.

E-mail: bgraham@nih.gov

Received 28 June 2006; revised and accepted 26 September 2006; published online 24 November 2006 immunoperoxidase method on paraffin-embedded tissue from autopsies before 1960, we have identified a group of such cases that are immunostainpositive for RSV antigen. The current study describes the morphological findings, inflammatory cell response, and viral distribution in these cases of naturally occurring RSV infection.

\section{Methods}

\section{Case Selection}

Autopsy logs were reviewed, beginning with the establishment of Vanderbilt Medical School in 1925, through 1959. Cases identified for further evaluation included those children 1 month to 3 years of age, who died between December 1 and March 1, with a major diagnosis of 'atypical pneumonia.' An additional criterion was a negative bacterial lung culture, a test performed routinely at post-mortem 
examination at that time. Autopsy reports were reviewed for the details of gross and microscopic morphology and clinical history. Final case selection consisted of cases with histopathological features (as described in the autopsy reports) that included bronchiolitis with or without diffuse interstitial inflammation, and in which the clinical features were compatible with RSV infection, including rhinorrhea and cough combined with coryza, wheezing, or apnea. A total of 11 cases meeting these criteria were identified.

The index case of acute untreated infection was an infant who died in a motor vehicle accident 1 day after RSV antigen detection in a throat swab (Case 4); this case differs from the archival cases in that death was not attributable to RSV infection, and there were only three symptomatic days before death.

\section{Control Selection}

Negative control cases were selected by the criteria described above, except that the date of death was in June through August.

\section{Preparation of Interpretable Slides}

The original autopsy slides were retrieved and found to be suboptimal for detailed examination due to fading of the original hematoxylin and eosin stain, with loss of morphological detail. While limited information about storage history is available, paraffin blocks were known to have been stored in nontemperature-controlled conditions for a number of years. In several cases, the paraffin blocks had melted together and required re-embedding. Cases from the 1920s to 1940s were embedded in a low melting point paraffin, and possibly beeswax (cases from 1920s). Historically, the most likely fixative was a mercury-based fixative, probably Zenker's.

Two lung blocks per case had been archived. From each block, multiple 3-5 $\mu$ m sections were prepared. Routine hematoxylin and eosin- and PAS-stained slides were prepared by standard methods,${ }^{8}$ including a mercury-binding step to prevent precipitation of any residual mercury salts from Zenker's fixative.

\section{Immunohistochemistry}

All studies were performed on paraffin-embedded tissue. Antibodies used are commercially available and are listed in Table 1. RSV immunostaining was performed manually. Sections were cut at $5 \mu \mathrm{m}$ thickness onto positively charged slides. After routine deparaffinization, the sections were placed in citrate buffer ( $\mathrm{pH}$ 6.0) microwaved for $10 \mathrm{~min}$, allowed to cool for $20 \mathrm{~min}$, and then placed in phosphate-buffered saline (PBS). Nonspecific binding was blocked by incubation with Dako (Glostrup,
Table 1 Reagents used for immunohistochemistry

\begin{tabular}{llll}
\hline Antibody specificity & Company & Clone & Titer \\
\hline RSV & Biodesign & Polyclonal; & $1: 250$ \\
& International & biotin-conjugated & \\
CD3 & Dako & UCHT1 & $1: 100$ \\
CD20 & Dako & L26 & $1: 20$ \\
CD4 & Novocastra & 1F6 & $1: 5$ \\
CD8 & Dako & C8/144B & $1: 5$ \\
CD68 & Dako & KP1 & $1: 100$ \\
CD1a & Dako & O10 & $1: 100$ \\
Von Willebrand Factor & Dako & F8/86 & $1: 50$ \\
\hline
\end{tabular}

${ }^{a}$ All slides pretreated with microwave heating for $10 \mathrm{~min}$ in citrate buffer, pH 6.0.

Denmark) serum-free protein block (Dako \#X0909). The sections were then incubated with polyclonal biotin-conjugated goat anti-RSV (Table 1) for $2 \mathrm{~h}$ at room temperature, and washed in PBS. Appropriately diluted peroxidase-conjugated streptavidin was added for $30 \mathrm{~min}$. After three PBS washes, the color was developed with diaminobenzidine (DAB), and a hematoxylin counterstain was applied.

Stains for CD3, CD20, CD68, CD4, CD1a, CD8, and factor VIII (Table 1) were performed on a Ventana automated immunostainer (Ventana Automated ES, Ventana Medical Systems Inc., Tucson, AZ, USA). Rabbit secondary antibody was applied, and the DAB chromogen developed following kit instructions (Vectastain Kit, Vector Laboratories, Burlingame, CA, USA, cat\# PK6106). Immunostaining for cellular phenotypes was only possible for the tissue from the index case and was not achieved for the archival cases.

Immunohistochemical staining was interpreted as positive or negative; the lineage of positive cells was identified by anatomic location and cytological features.

\section{Results}

\section{Case Selection and Clinical Features}

Eleven archival cases met all criteria; three of these were positive for RSV antigen by immunohistochemistry, as was the index case. All four control cases were immunostain-negative for RSV antigen.

A summary of the clinical data from the four cases is presented in Table 2. Patient ages ranged from 1 month to 3 years. The three youngest patients had significant congenital comorbidities. The 3-year-old (Case 2) had a relatively recent history of pertussis and measles infections, and had a longer clinical course than the other three.

\section{Immunohistochemical Staining for RSV Antigen}

Crisp staining for RSV antigen was observed in the bronchiolar and bronchial epithelial cells (Figure 
1a). The smallest airways often had circumferential staining, while the larger airways most often showed positivity in clusters of three to five cells at the luminal surface; the small cuboidal basal cells were routinely immunostain-negative (Figure 1b). The antigen staining was concentrated at the surface,

Table 2 Clinical features of identified cases

\begin{tabular}{|c|c|c|c|}
\hline $\begin{array}{l}\text { Case number } \\
\text { and year }\end{array}$ & $\begin{array}{l}\text { Age and } \\
\text { gender }\end{array}$ & $\begin{array}{l}\text { Duration of } \\
\text { symptoms }\end{array}$ & Coexisting conditions \\
\hline 1 & 1 month & 2 days & Congenital syphilis: bone \\
\hline 1931 & & & $\begin{array}{l}\text { lesions and positive } \\
\text { Wasserman test }\end{array}$ \\
\hline 2 & 3 years & 10 days & Pertussis 5 weeks before \\
\hline 1946 & $\mathrm{~F}^{\circ}$ & & $\begin{array}{l}\text { admission } \\
\text { Measles } 3 \text { weeks before } \\
\text { admission }\end{array}$ \\
\hline 3 & 1 month & 4 days & Intrapartum hypoxia with \\
\hline 1949 & & & $\begin{array}{l}\text { brain injury } \\
\text { Neonatal hyperglycemia }\end{array}$ \\
\hline $\begin{array}{l}4 \\
1999\end{array}$ & $\begin{array}{l}15 \text { months } \\
\mathrm{M}\end{array}$ & $36 \mathrm{~h}$ & $\begin{array}{l}\text { Complex congenital heart } \\
\text { disease }\end{array}$ \\
\hline
\end{tabular}
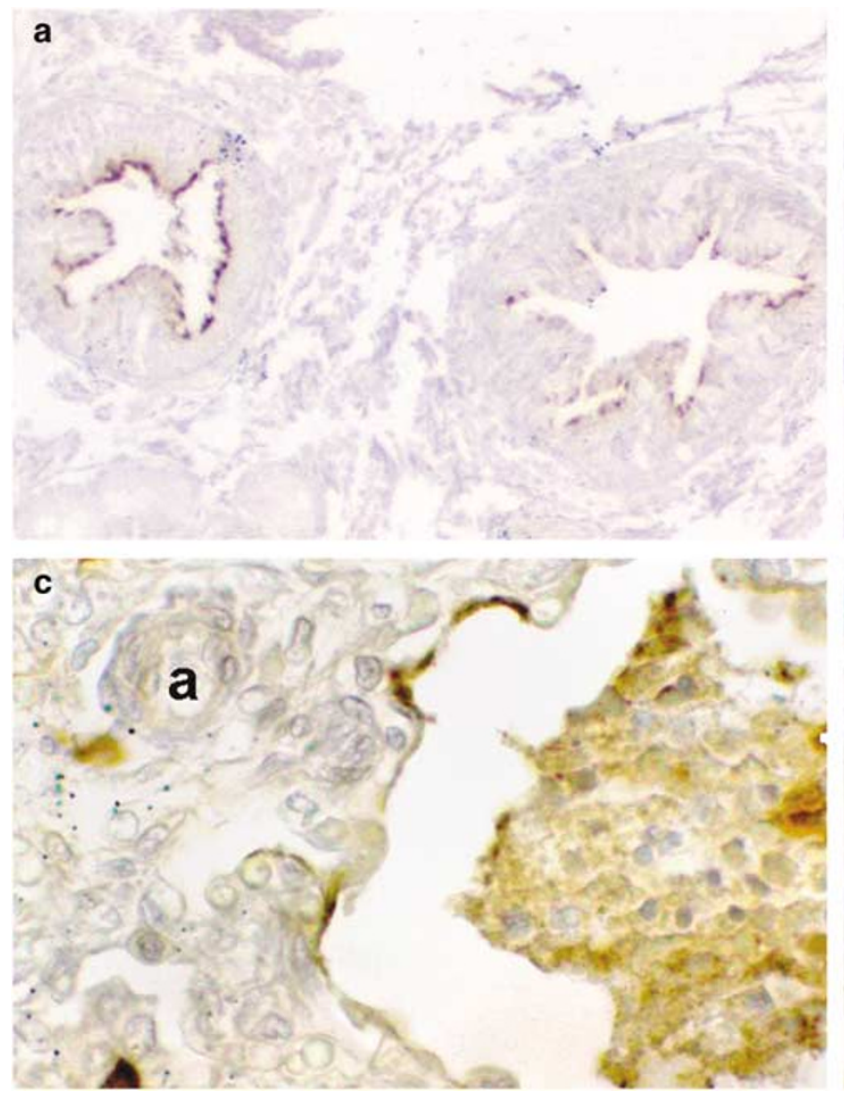

and lateral aspects of the columnar cells and some of the immunostain-positive epithelial cells could be clearly identified as ciliated (Figure 1b). The intraluminal debris within airways was often positive for RSV antigen as well (Figure 1c), including the cytoplasm of intrabronchiolar macrophages. The intrabronchiolar syncytia observed in the lung tissue of patient 3 stained for RSV antigen in a membrane distribution (Figure 1d).

At the alveolar level, immunostain-positivity was observed in a continuous linear fashion along the alveolar surfaces in samples from patients 3 and 4 , with cells typical of both type 1 and 2 pneumocytes staining positively (Figure 2a). Intra-alveolar exudates also contained RSV antigen, both within the cytoplasm of macrophages and in a nonspecific debris-staining pattern (Figure 2b).

\section{Bronchiolitis and Airway Histology}

Acute bronchiolitis (Figure 3a) was prominent in all cases, involving medium and small bronchioles most severely (150 $\mu \mathrm{m}$ and smaller); but proximal
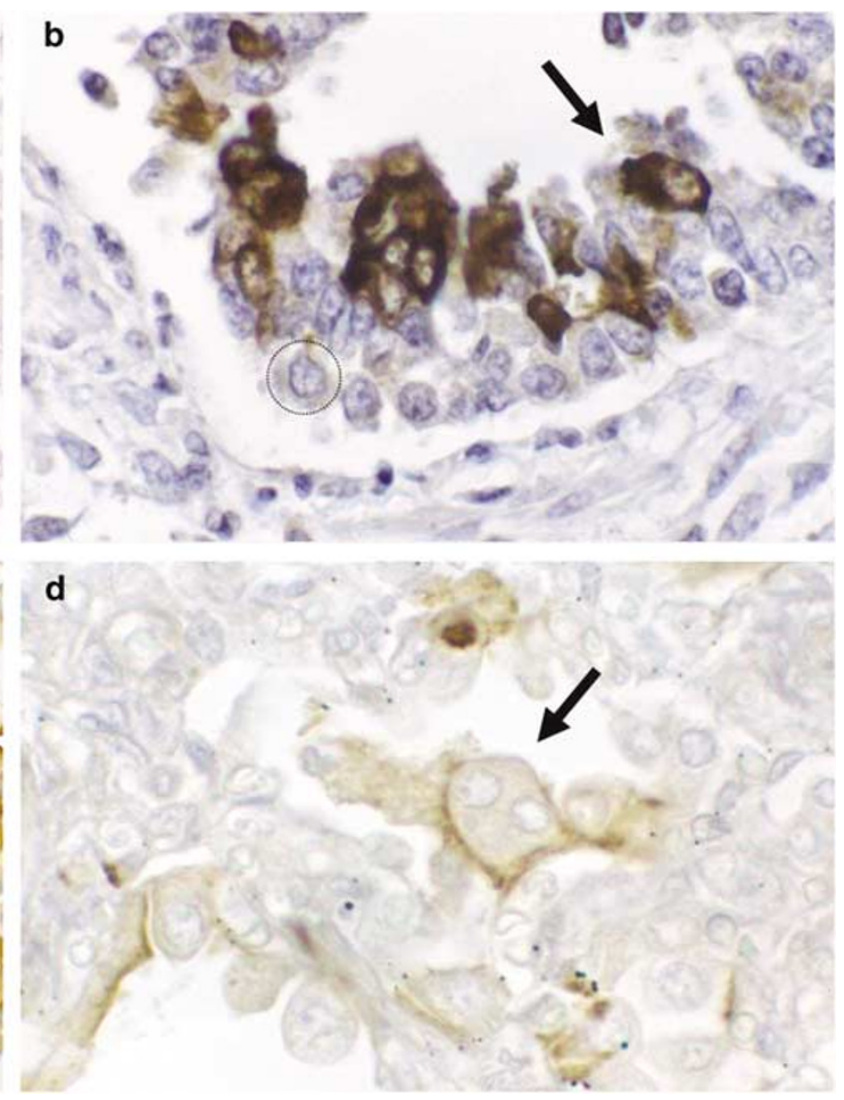

Figure 1 Bronchiolar localization of RSV antigen. Medium-sized muscular bronchioles demonstrate RSV antigen circumferentially and limited to surface epithelium in 1931 case (a). Bronchiolar epithelium demonstrating RSV antigen in surface epithelium, basal epithelium is antigen-negative, and ciliated (arrow) cells are clearly infected. It is likely that nonciliated cells are also infected because of the near circumferential staining pattern (a), and it is equally clear that the basal progenitor cells (circled) are not infected in 1999 case (b). Intraluminal debris in small airways, including epithelium and inflammatory cells, stains positively for RSV antigen. Arteriole ('a') in 1949 case (c). Epithelial syncytia (arrow) express RSV antigen in a membrane distribution in 1949 case (d). Original magnifications $\times 25$ (a), $\times 250(\mathbf{b}-\mathbf{d})$. 

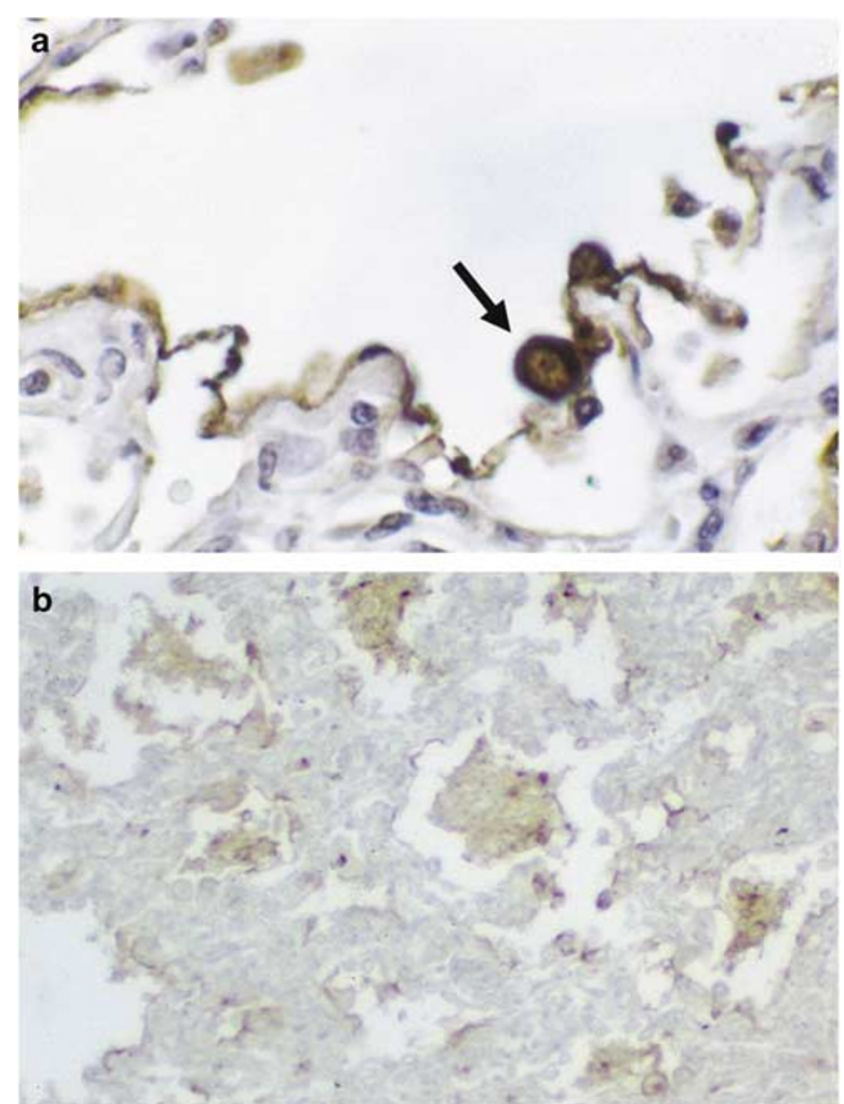

Figure 2 Alveolar localization of RSV antigen. Linear staining for RSV antigen in alveoli demonstrates that both type 1 and 2 (arrow) pneumocytes are infected in the 1999 case (a). Airway and alveolar lumens are filled with RSV antigen-positive debris in the 1949 case $(\mathbf{b})$. Original magnifications $\times 250(\mathbf{a}), \times 62.5(\mathbf{b})$.

bronchioles and cartilaginous airways were also involved. Most airway lumens were occluded by debris composed of sloughed epithelial cells and macrophages, with admixed fibrin and a minor component of mucin; remaining adherent epithelial cells contained only rare minimal cytoplasmic mucin (Figure 3b). Epithelial sloughing observed in cross-section was not typically circumferential, but rather, patchy. Numerous intrabronchiolar syncytia were present in the lungs of patient 3 (Figure 3c), but were not seen in the other cases. However, papillary projections of epithelium without syncytia were sometime noted in both this case and other cases and may contribute to airway narrowing.

The major locus of bronchiolar inflammation was in the submucosa, deep to the muscularis layer and was sometimes accompanied by large hyperplastic lymphoid aggregates (Figure 4a). These collections of inflammatory cells were centered on bronchial arteries, which supply the bronchial wall down to the level of terminal bronchioles in humans (Figure 4b). These arteries were often prominently dilated and congested. In the smallest inflamed airways, which lack mural bronchial vessels, the bronchovascular inflammation was demonstrably centered on the accompanying pulmonary artery branch
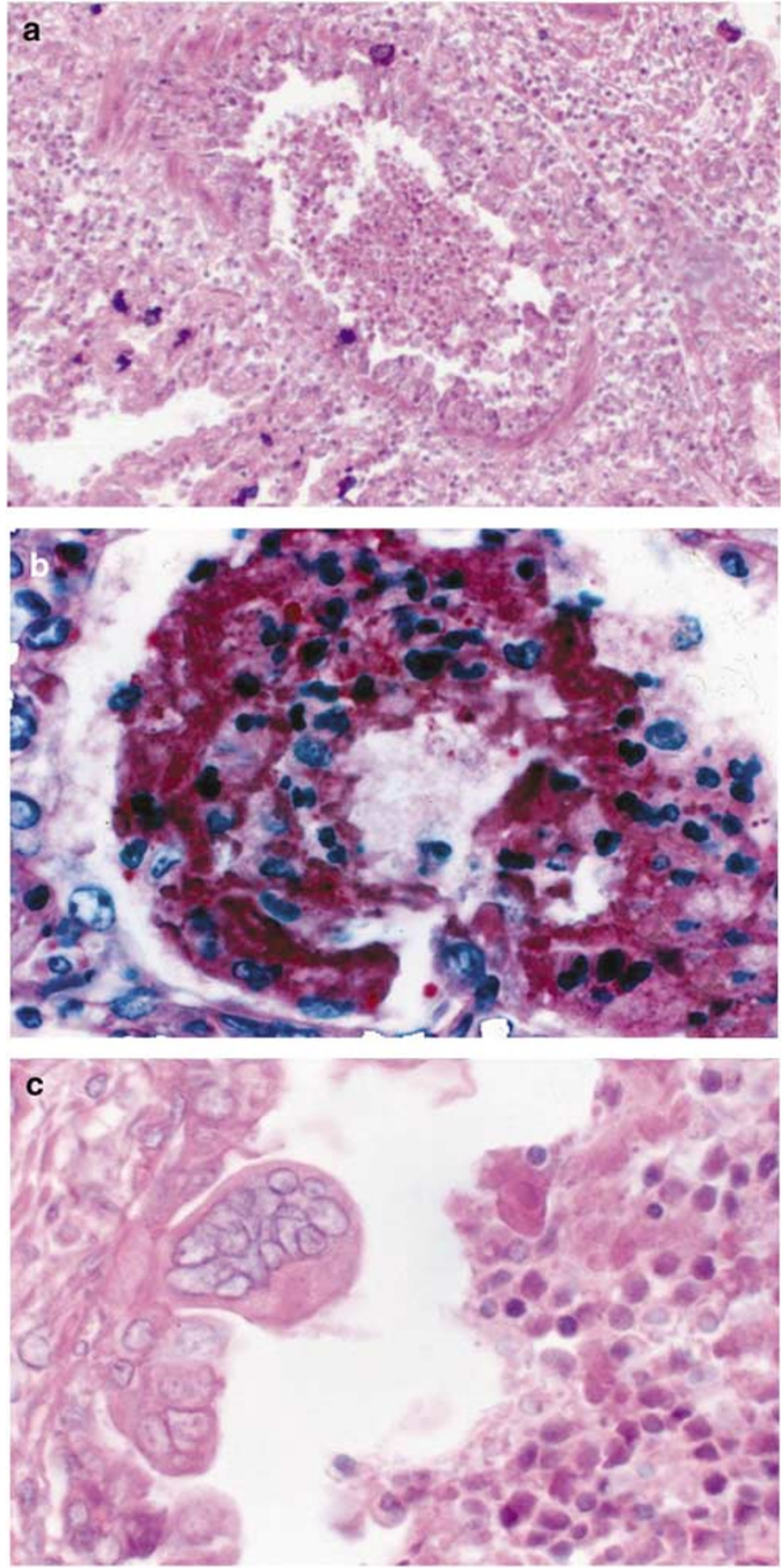

Figure 3 Histopathological features of bronchiolar inflammation Acute bronchiolitis in medium-sized airway with intraluminal cell fragments composed of dead epithelium and inflammatory cells, and amorphous debris in 1931 case (a). Intraluminal debris includes mucus, fibrin, epithelial cells, and inflammatory cells in 1949 case (b) (dPAS stain). Intrabronchiolar syncytia are present adjacent to intraluminal cellular debris in 1949 case (c). Original magnifications $\times 62.5(\mathbf{a}), \times 250(\mathbf{b}$ and $\mathbf{c})$.

(Figure 4c). Therefore, the pattern of the inflammatory infiltrate around an airway appears to be determined by the distribution of arterioles adjacent to the airway. Inflammatory infiltrates are symmetric and circumferential in the small bronchioles and larger airways, but at the level of the terminal bronchioles it is often asymmetric and more clearly centered around the pulmonary arteriole (Figure 4d). 

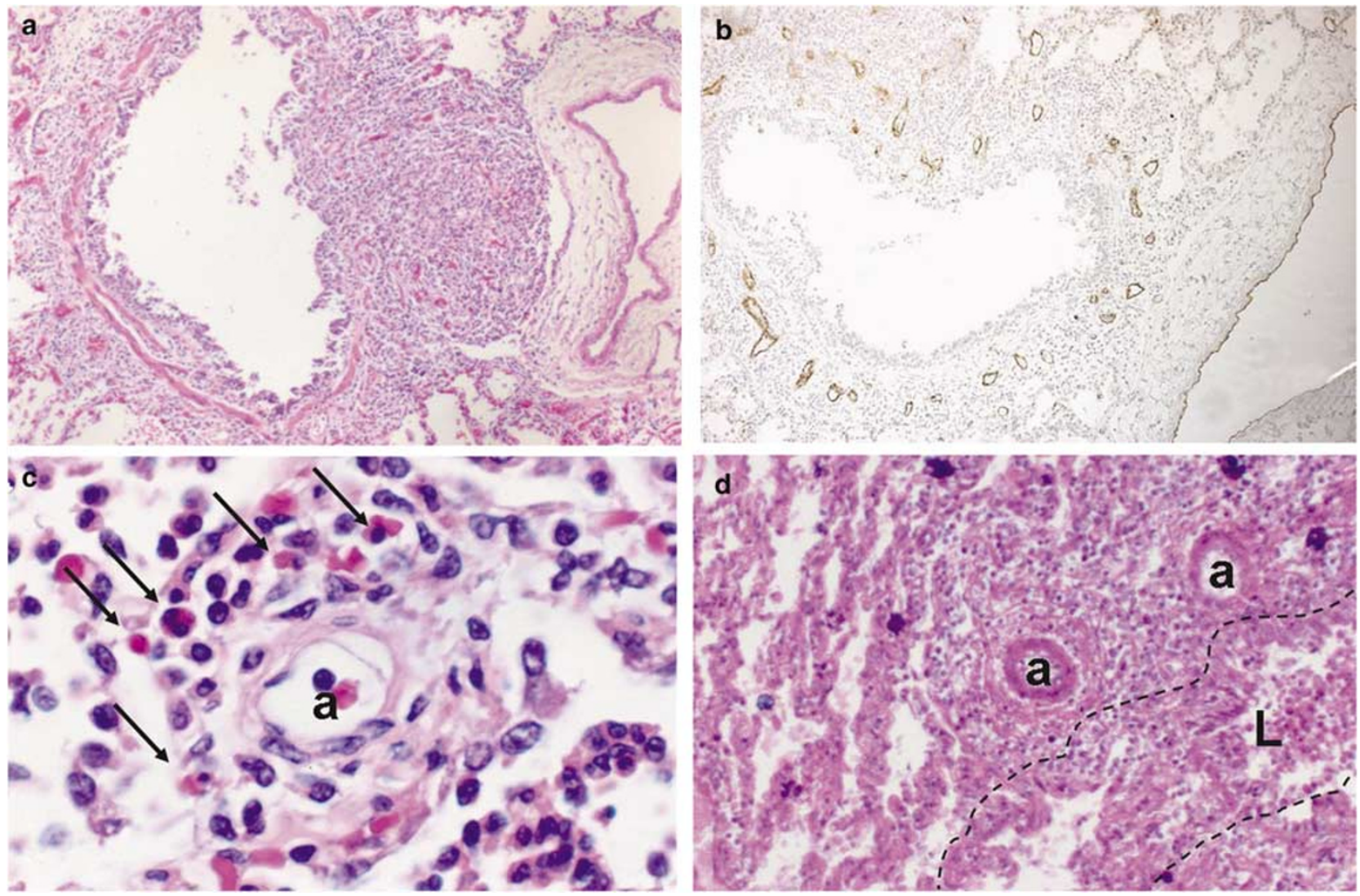

Figure 4 Vasocentric inflammation determines the pattern of bronchiolitis. A large lymphoid aggregate lies between a pulmonary artery and a large bronchiole. The bronchiole is edematous and is surrounded by a lymphocytic infiltrate, which is predominantly outside the muscularis; the submucosa is mildly expanded by edema and inflammation. The bronchial arteries around the airway are congested in the 1999 case (a). Factor 8 labeling demonstrates the distribution of bronchial arteries around the larger airways; the larger pulmonary artery is at right. This distribution of bronchial arteries allows vasocentric inflammation to circumferentially surround large- and medium-sized airways in the 1999 case (b). Inflammation is centered on a bronchial artery and includes both mononuclear cells and occasional eosinophils (arrows) in 1999 case (c). It is clear that in the smallest airways (indicated by 'L' for lumen and demarcated by dashed lines), the inflammation is centered on the pulmonary arteries (a) and not the airway in the 1931 case (d). Original magnifications $\times 10$ (a and $\mathbf{b}), \times 40(\mathbf{c}$ and $\mathbf{d})$.
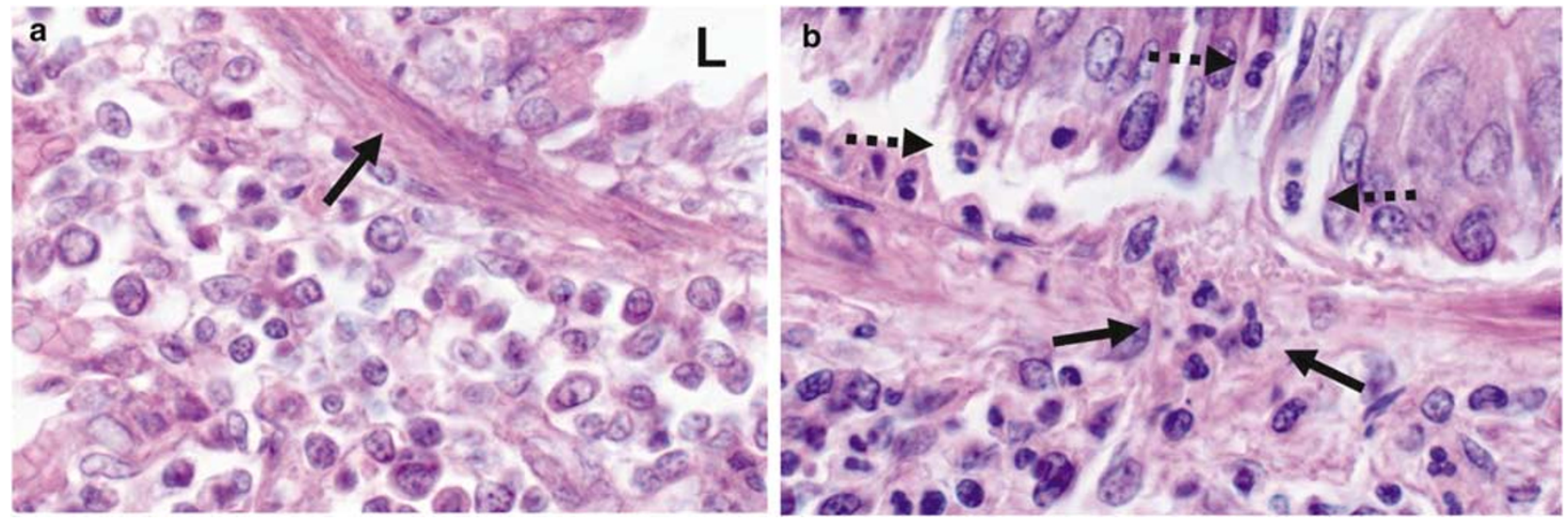

Figure 5 Relative segregation of mononuclear cells and polymorphonuclear leukocytes in airway. Peribronchiolar mononuclear inflammatory infiltrate is primarily submuscular, and is peripheral to the smooth muscle (arrow). 'L' indicates the airway lumen in the 1931 case (a). While neutrophils are commonly found in BAL, the predominant cells in the peribronchiolar tissues are mononuclear. Neutrophils can sometimes be found migrating across gaps in the muscularis (arrow) toward the airway lumen and interspersed within the epithelium (dashed arrows) in 1946 case (b). Original magnification $\times 250$ (a and b).

Within these peribronchiolar infiltrates, deep to the muscularis, the predominant leukocyte was mononuclear, cytologically typical of macrophages and small or transformed lymphocytes (Figure 5a). A minority neutrophil component (10-25\%) was observed in the submucosa, and if present, it was 
typically on the adluminal aspect of the bronchial artery; occasional neutrophils were seen crossing the muscularis or present within the basal aspect of the epithelium, along the basement membrane (Figure $5 b)$. Intact neutrophils were not routinely observed free in the bronchiolar lumens to any significant extent. Eosinophils were occasionally observed in peribronchiolar infiltrates (Figure 4c), but were not a dominant component of the inflammatory process.

\section{Lung Parenchymal Histology}

Interstitial inflammation was present in the lungs of all four patients (Figure 6a) and was associated with marked capillary congestion. Three patients also had intra-alveolar leakage of fibrin and mononuclear cells, and occasional neutrophils (Figure 6b); however, fibrin and macrophages generally predominated in the airspace infiltrates (Figure 6a and c).
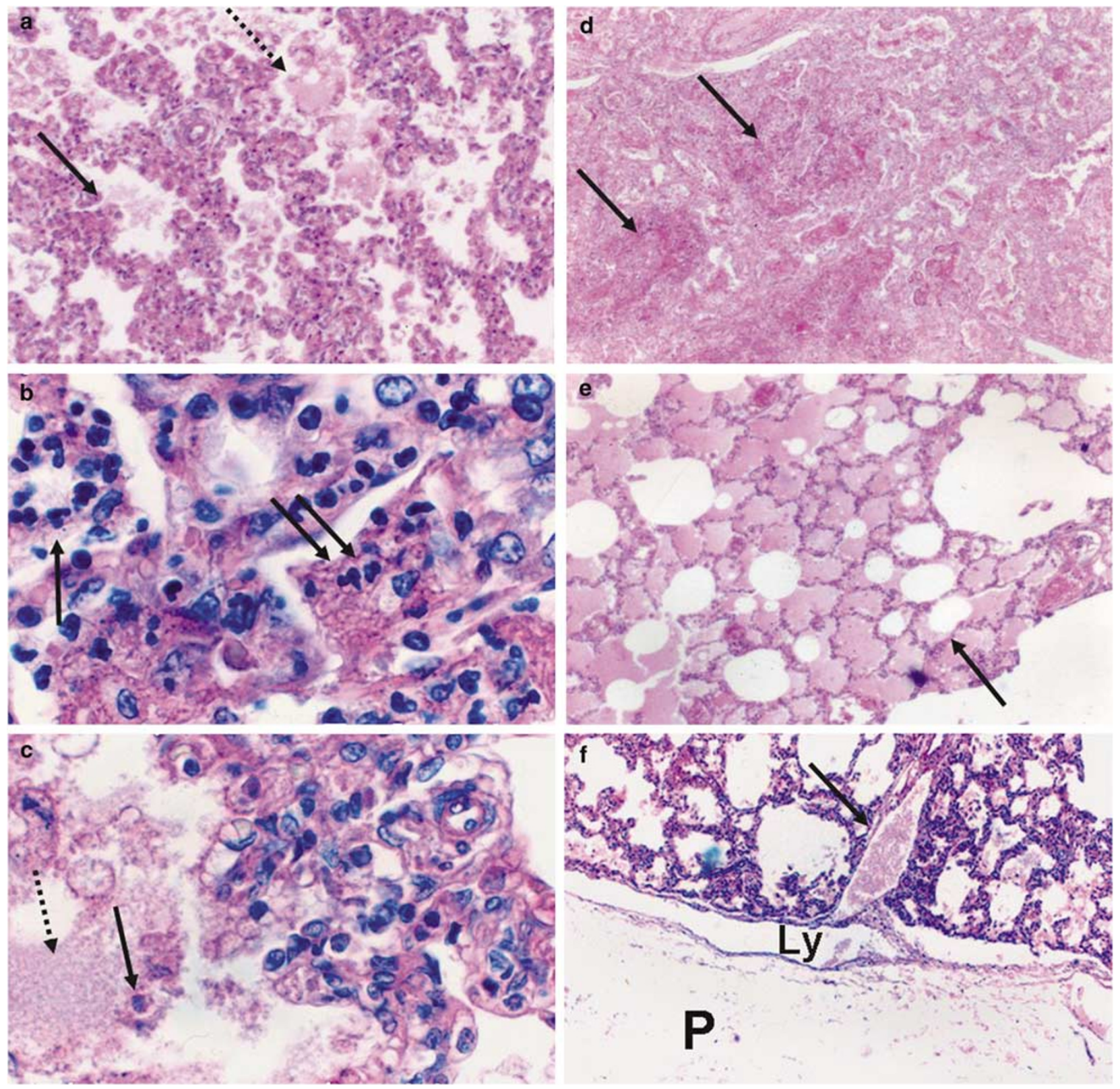

Figure 6 Airway obstruction and edema contribute to compromise of lung function. The lung parenchyma shows interstitial mononuclear infiltrates, capillary congestion (arrow), and granular intra-alveolar edema fluid (dashed arrow) in 1931 case (a). Occasional neutrophils (arrows) are found in the alveolar exudates in 1931 case (b). Granular fibrinous debris (dashed arrow) and macrophages (arrow) are the major components of the alveolar exudates in the 1931 case (c). In some cases, there is frank alveolar hemorrhage and necrosis (arrows). The basis for this is unknown, and, although no bacteria were reported, it is possible that this finding is the result of a mixed infection in the 1949 case (d). Watery alveolar edema was present and appears to be more serous with an air-fluid meniscus occasionally demonstrable (arrow) possibly influenced by a recent pertussis infection in the 1946 case (e). The pleura (P) is edematous and the subpleural lymphatic (Ly) is dilated. The septal lymphatic (arrow) is also dilated and full of lymph. These features indicate increased interstitial lymph flow associated with pulmonary edema in the 1931 case (f). Original magnifications $\times 62.5$ (a and f), $\times 250$ (b and $\mathbf{c}), \times 25$ (d and $\mathbf{e})$. 
Patient 3 manifested the most severe pneumonitis, with rare foci of necrosis and hemorrhage (Figure 6d). Patient 2 had a unique finding of diffuse pulmonary edema present in areas uninvolved by bronchiolitis and pneumonitis (Figure 6e). The pleura was consistently affected by moderate edema and dilated lymphatics (Figure 6f), and sometimes septal lymphatics were dilated and full of lymph. The pulmonary veins were not a focus of inflammation.

\section{Bronchiolar-Associated Lymphoid Tissue Histology}

Striking hyperplasia of the bronchiolar-associated lymphoid tissue (BALT) was consistently observed, present in the notch between the bronchiole and its companion artery (Figures 4a and 7a). These nodules, often quite hyperplastic, resembled lymph node follicles, and in the index Case 4 were consistently composed virtually entirely of CD20 + cells (Figure 7a). Occasional BALT nodules protruded into the bronchiolar lumens (Figure $7 \mathrm{~b}-\mathrm{d}$ ). The lymphoid aggregates were again primarily composed of $\mathrm{CD} 20+\mathrm{B}$ cells in Case 4, but occasional CD3 + T cells (Figure 7c) and CD68 + monocytes (Figure $7 \mathrm{~d}$ ) were also present.

\section{T-Lymphocyte Response to RSV in Lung}

CD3 + T-lymphocytes are critical for the adaptive immune response to most virus infections. Immunostained sections from patient 4, the 1999 case, recently diagnosed and being treated with supportive care as an outpatient reveals a significant CD3 + T-cell infiltration in lung (Figure 8a). They are in the greatest abundance in the space between pulmonary arterioles and small distal airways (Figure 8a), and there are occasional intraepithelial CD3 $+\mathrm{T}$ cells observed (Figure 8b) creating the impression that there is a chemotactic gradient established by the
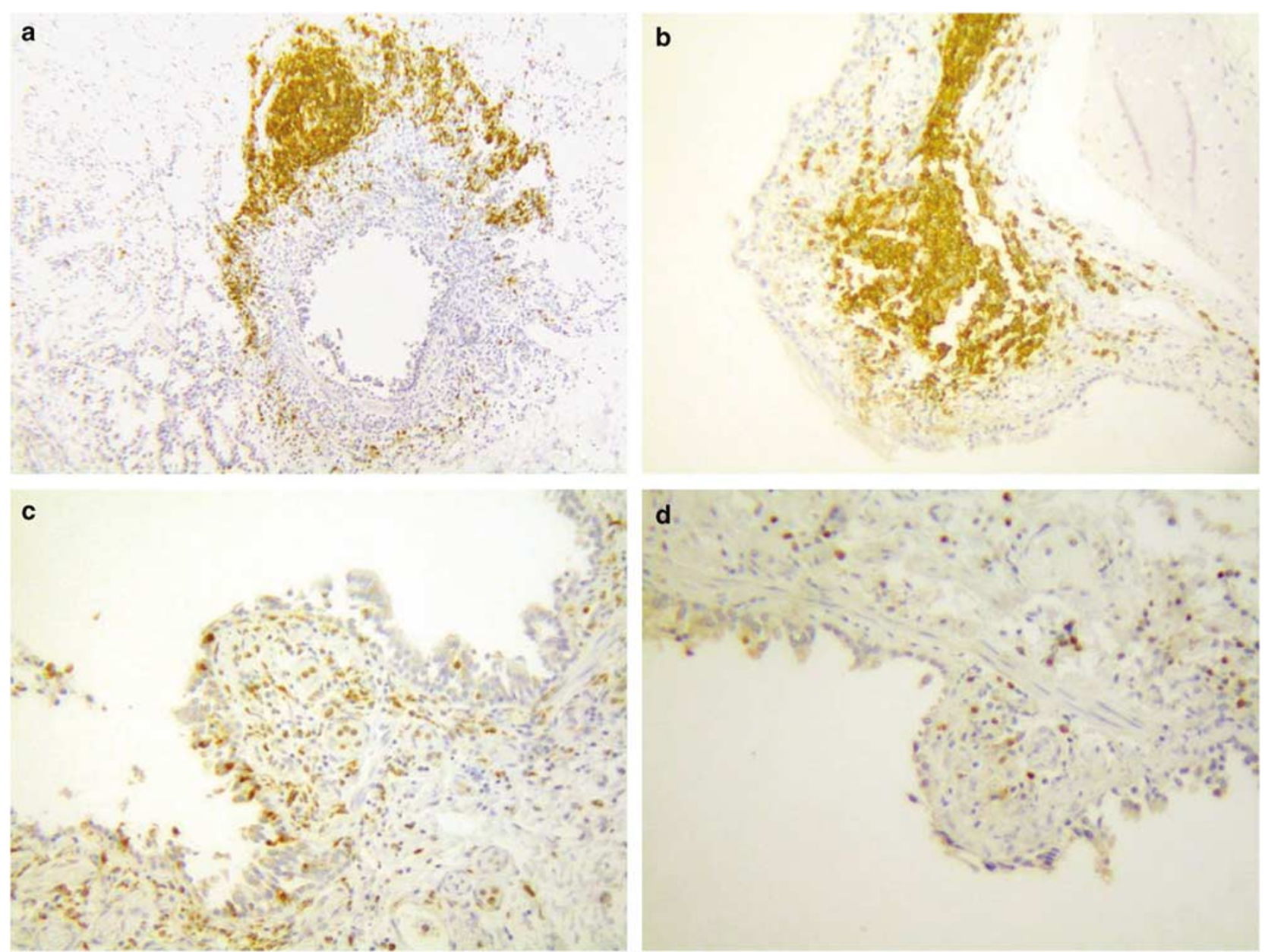

Figure 7 Peribronchial and intraluminal lymphoid aggregates. Peribronchial lymphoid aggregates are primarily composed of CD20 + B lymphocytes, presumably part of the BALT. During the response to RSV infection, some areas appear to develop the appearance of a hyperplastic lymph node follicle (a). The hyperplastic lymphoid nodules, which extend into and partially obstruct the airway lumen, are also predominantly composed of CD20 + B lymphocytes (b). Occasional CD3 + lymphocytes are present in the hyperplastic nodules (c). CD68 + monocytes are sparse in hyperplastic nodules (d). All images are from the 1999 case; original magnifications $\times 25$ (a), $\times 62.5(\mathbf{b}-\mathbf{d})$. 

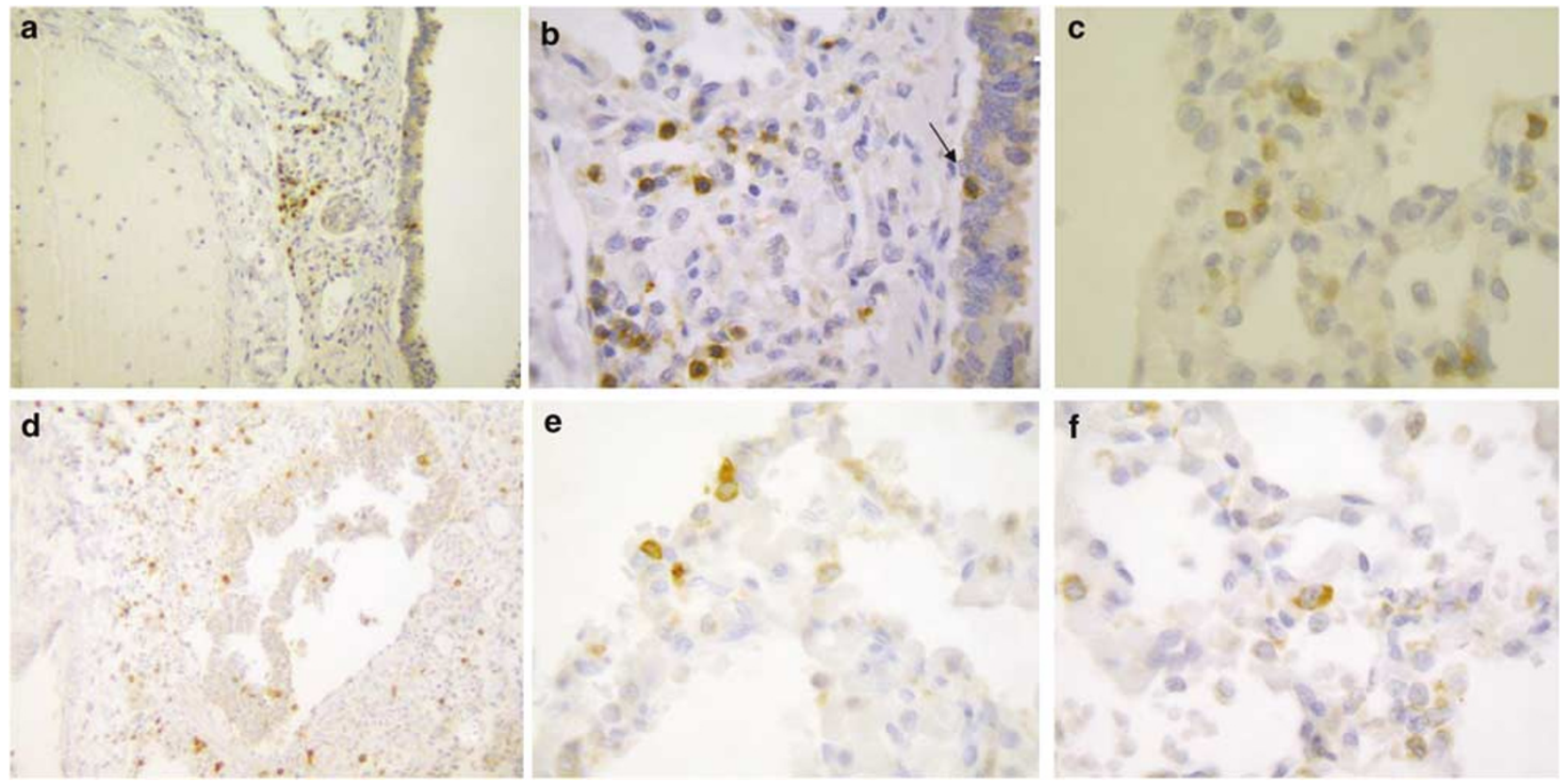

Figure 8 T-lymphocytes contribute to the inflammation of acute RSV infection. CD3 + T-lymphocytes are a significant component of the submuscular inflammatory infiltrate around small bronchioles. They are seen primarily between the pulmonary arteries and small bronchioles in the distal airways (a). They are occasionally seen within the basal cell layer of the bronchiolar epithelium (b). There is also a prominent inflammatory cell infiltrate in the alveolar interstitium (c). The majority of T cells in the peribronchiolar infiltrates (d) and in the alveolar interstitium (e) are CD8 + . CD4 + cells are rarely found in the peribronchiolar infiltrates and even more rarely at the level of the alveoli (f). All images are from the 1999 case; original magnifications $\times 25$ (a and d), $\times 62.5(\mathbf{b}), \times 250(\mathbf{c}-\mathbf{f})$.

RSV-infected airway epithelium. This is also suggested by the finding that the intraepithelial CD3 + cells appear to express CD20 indicating their high state of activation (data not shown). CD3 + T cells are also present in the alveolar interstitium (Figure 8c). In both the peribronchiolar and interstitial infiltrates, CD8 + T cells (Figure 8d and e) significantly outnumber CD4 $+\mathrm{T}$ cells (Figure 8f), but there are more CD3 + T cells present than the sum of $\mathrm{CD} 8+$ and CD4 $+\mathrm{T}$ cells. This finding suggests that there may be a substantial CD3 + double-negative T-cell population in the inflammatory response to RSV.

\section{Monocytes, Macrophages, and Dendritic Cells in RSV-Infected Lung}

The interstitial and intra-alveolar mononuclear infiltrates had a much greater abundance of CD68+ cells than CD3 + or other cell types in Case 4 (Figure 9). These cells are particularly abundant in the peribronchiolar submucosal infiltrates and among the cells that penetrate to the airway epithelium (Figure 9a and b). They are in highest concentration in the region between pulmonary vessels and small airways or in the connective tissues and BALT at the notch of branching airways (Figure 9b and c). Distally, the CD68 + cells (alveolar macrophages) are also abundant but confined to the alveolar spaces.
CD1a + dendritic cells (DCs) were rarely detected in airway epithelium (Figure 10a) or in BALT near airway branches in Case 4 (Figure 10b). While DCs could still potentially be the functionally dominant antigen presenting cell (APC) and their low number may be explained by their movement into lymph nodes during the early inflammatory process, it should be noted that they represent an extremely minor fraction of the professional APCs present in the lung infiltrates.

\section{Discussion}

RSV is an important cause of lower respiratory disease in infants, with a high priority for vaccine development. Despite its importance and extensive characterization of pathology in animal models, there are very few descriptions of the human pathology of RSV infection in infants that are not confounded by modern treatment interventions. Therefore, we searched the Vanderbilt University Pathology archives and confirmed three cases of RSV infection in infants who died during winter months with bronchiolitis before 1960. In addition, we evaluated a contemporary case in which the infant died 2 days after becoming symptomatic with a confirmed RSV infection. The archival cases required re-embedding before processing. Nevertheless, RSV immunostaining was able to confirm infection in tissue that was more than 65 years old. 

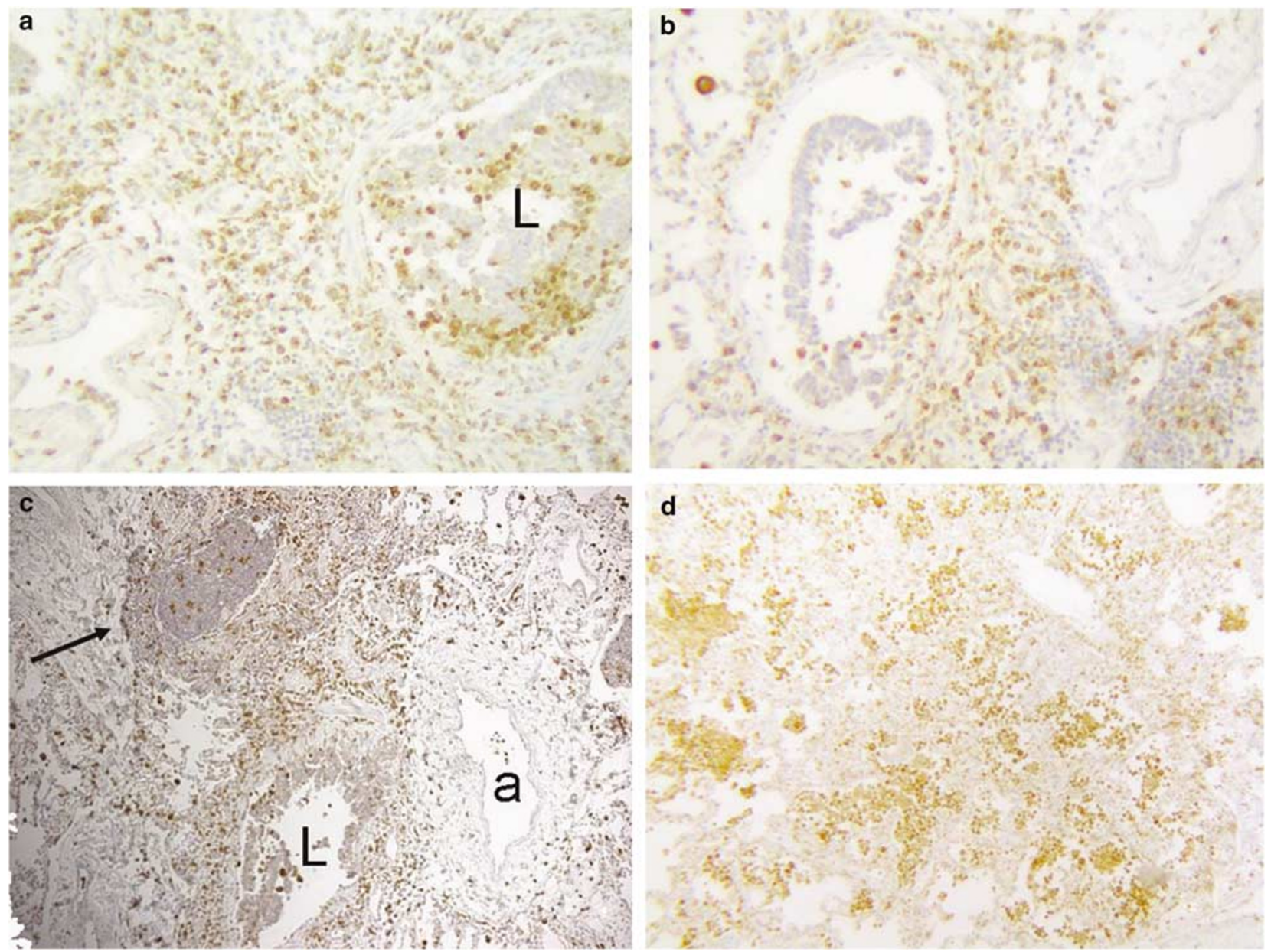

Figure 9 Macrophages are the predominant cell types in both the peribronchial and alveolar inflammatory infiltrates. The majority of cells in the infiltrates around airways are CD68 + macrophages, which are also prominent in the intraluminal (L) debris (a). CD68 + macrophages are also present within the bronchiolar epithelium (b). CD68 + macrophages are prominent in the bronchovascular connective tissue, particularly between arteries and branching airways where they are also within the follicle-like expanded BALT (arrow) (c). In the distal lung parenchyma most of the CD68 + cells are alveolar macrophages present in the alveolar spaces and not in the alveolar interstitium (d). All images are from the 1999 case; original magnifications $\times 62.5$ (a and b), $\times 25$ (c and d).
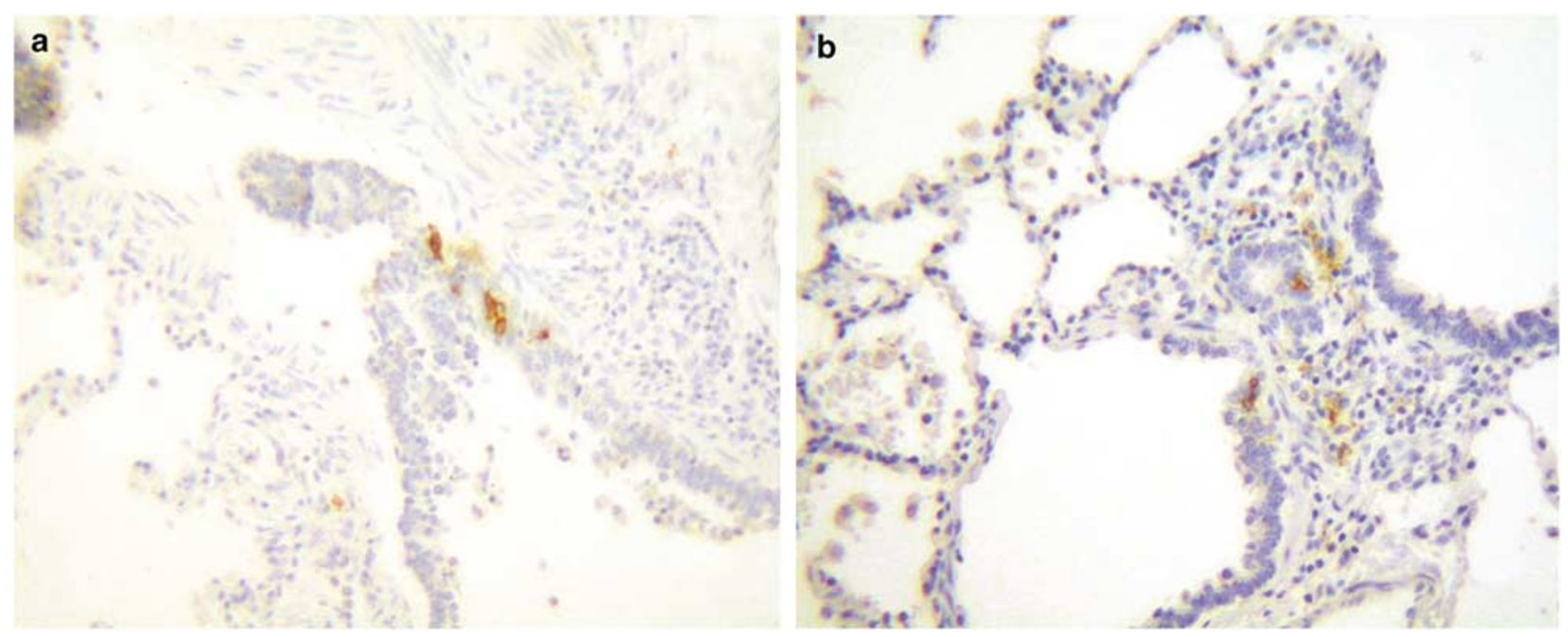

Figure 10 DCs in RSV-infected lung. CD1a + cells can occasionally be found in the bronchiolar epithelium (a) and in the peribronchiolar connective tissue (b). All images are from the 1999 case; original magnification $\times 62.5$ (a and b). 
Although Dr Ernest Goodpasture described cases of virus pneumonia consistent with RSV infection in $1939,{ }^{9}$ our case from 1931 is the earliest confirmed case of RSV infection ever reported. Unfortunately, attempts to extract viral RNA from the archival samples for amplification and sequencing as recently achieved for the 1918 strain of influenza ${ }^{10}$ were unsuccessful.

The virus was found in airway epithelial cells in all four cases, and two cases also had evidence of RSV-infected alveolar epithelium. This is consistent with the distribution of virus-infected cells in reports of the pathology of bovine RSV in calves, ${ }^{11}$ and indicates that lower respiratory disease from RSV infection is not just an indirect effect of infection in the bronchiolar epithelium, but reflects direct virus infection of the alveolar epithelium. The finding of extensive virus infection in both the small airways and the alveolar epithelium in patient 3 who was recently diagnosed and being managed as an outpatient is particularly enlightening. It provides direct evidence that RSV can be a lower respiratory tract infection even when symptoms are primarily restricted to the upper respiratory tract and with illness that is considered to be relatively mild. This has been suggested by the clinical evaluation of RSV-infected outpatients ${ }^{12}$ and suggested in another autopsy series. ${ }^{6}$ The distribution of virus-infected cells ranged from clusters of cells in large cartilaginous airways to more circumferential infection of epithelium in the smallest airways. This suggests a tropism for cells of the distal airway, and more homogeneous cell populations in lower airways. As expected, ${ }^{13}$ ciliated cells are infected, and it appears that nonciliated cells are also infected as noted in an adenoid organ culture model. ${ }^{14}$ Basal cells appear to distinctly be not infected (Figure 1a). This may provide a cellular basis for transitioning to mucus metaplasia ${ }^{15}$ that can occur in the RSVinfected airway, and suggests that basal cells have innate protection from RSV that allows restoration of the airway. This may be related to the apical budding and entry of RSV from polarized epithelial cells. ${ }^{16}$

In the alveoli, the dominant cell type infected was the type 1 pneumocyte. Although some cuboidal cells were immunostain-positive, it is difficult to know whether these represented terminally differentiated type 2 pneumocytes or reparative type 2 cells. While infection of the alveolar epithelium was patchy, in areas of infection the immunostainpositive cells were confluent, suggesting there was no selective cellular tropism in this area and suggests that virus was able to efficiently spread from cell to cell. Only one case demonstrated significant syncytium formation in the bronchiolar epithelium, and we did not appreciate any cytoplasmic inclusions. Most of the alveolar macrophages were immunostain-positive. Macrophages can be infected in cell culture, ${ }^{17}$ so this may represent actual infection, but it is also possible that this represents phagocytosed antigen. Although DCs can be infected with RSV in vitro ${ }^{18}$ and CD1a + cells were detected in peribronchiolar tissue, no RSV-positive cells were identified that appeared to have DC morphology. DCs are highly mobile and, if infected, may have already moved to areas of organized lymphoid tissue for induction of adaptive immune responses.

The cell surface determinants for defining lymphocyte subsets by immunocytochemistry were not as resilient to storage as the general tissue morphology and RSV viral antigens, and therefore this analysis was restricted only to the 1999 case. However, hematoxylin and eosin stains were available for all cases and showed similar histological features. Analysis of the cellular composition of bronchoalveolar lavage (BAL) fluid in acutely RSVinfected infants suggests that polymorphonuclear leukocytes play an important role in the response to acute RSV infection, at least in the airway and airspace compartments. ${ }^{19,20}$ This is consistent with finding high levels of IL-8 in respiratory secretions of RSV-infected infants ${ }^{21}$ or adults. ${ }^{22}$ In the current cases, debris consisting of sloughed epithelium and macrophages with fibrin and minimal mucin was noted to fill airway lumens. Neutrophils were not seen in the interstitial infiltrates, but were present as a minority cell type in the peribronchiolar tissue, with the dominant cell type being mononuclear. Both neutrophils and mononuclear cells were seen in the lumen. All cell types were present in the greatest density between the pulmonary or bronchiolar arteries and the airway lumen. This pattern was most characteristic of polymorphonuclear leukocytes suggesting that the chemotactic factors attracting them to migrate out of vessels are concentrated in the airway epithelium. In comparison, the distribution of mononuclear cells (both monocytes and lymphocytes) was broader suggesting that the factors produced by both airway epithelium and by cells in the lung parenchyma had chemotactic properties.

One purpose for better understanding the pathology of human RSV is to improve the understanding of how well animal models reflect the processes present in human disease. There are two aspects of inflammatory patterns in animal models of RSV that can be informed by findings from this study. In the murine model of RSV, the inflammatory infiltrate is centered on the arteriole rather than the bronchiole as it appears to be in humans. In mice, the bronchial arteries extend only to the larger airways, in contrast to human bronchial arteries that extend more distally to the smaller airways; therefore, in mice, inflammatory cells in all but the most proximal airways must come from pulmonary arterioles, which colocalize with the small airways, but do not surround them as bronchial arterioles like human bronchioles. The infiltrate in humans is centered on the bronchiolar arterioles even though it appears to be centered on the bronchiole, while in 
mice the infiltrate is clearly centered on pulmonary arterioles. A second feature of the pathology in rodent models of RSV is that there is a nearly exclusive mononuclear infiltrate during primary infection. ${ }^{23,24}$ This has been thought to be in conflict with the findings in human disease based on tracheal and bronchiolar lavage samples that show a predominance of polymorphonuclear leukocytes. The findings in this study suggest that there is a strong neutrophil movement to bronchiolar epithelium, while tissue infiltrates are predominantly mononuclear, which is consistent with patterns in the murine model.

The concentration of inflammatory cells in the space between the arterioles and the bronchioles is largely submuscular, but there were several examples of inflammatory cells moving through gaps in the smooth muscle surrounding the bronchioles (Figure 5b). This is a graphic demonstration of how products from immune cells can impact smooth muscle tone and affect airway hyperreactivity. It also suggests that if inflammation is not rapidly controlled more permanent disruption of these structures could occur during the remodeling process. Perhaps the most prominent findings were the variety of ways in which RSV was causing airway obstruction. In all the cases, small airways were seen that contained mixtures of inflammatory cells, fibrin, edema and mucus (Figure 6). In some areas, the infiltrates around the bronchovascular bundles were so dense that they resembled hyperplastic lymph node follicles (Figure 7), and, in other areas (Figures 4a, 8d, 9a-c, and 10b), cells were more loosely organized but still had a mixture of cell types that suggested that the collection of cells could be acting as an inductive site for immune responses. Both the hyperplastic follicles (Figure 7) and the peribronchiolar centers of inflammation (Figure 4a) contribute to airway narrowing or obstruction. Therefore, in addition to the direct consequences of immune effectors and airway hyperreactivity that are hallmarks of RSV pathogenesis, mechanical obstruction of the small airways appears to be playing a significant role in the pathogenesis of RSV disease.

A majority of cells around the bronchioles and in the alveolar interstitium are CD68 + cells and represent both alveolar macrophages and recruited monocytes from the arteries and capillaries. These cells are a rich source of many soluble factors and their role in RSV clearance and in the regulation of subsequent immune responses deserves further study.

CD3 + lymphocytes were the second most prevalent cells around the bronchioles, and interestingly the majority are double-negative. Further definition of this cell population may be important for understanding RSV pathogenesis. Possible explanations for this population include doublenegative T-regulatory cells (DN Tregs) ${ }^{25}$ that have not been studied in RSV infection; NK T cells that have been noted in the murine model of RSV; ${ }^{26}$ or $\gamma \delta$ T cells that have not been identified in lungs of RSV-infected mice $^{27}$ or BRSV-infected cattle. ${ }^{28}$ CD3 + CD8 + cells were also present, particularly in the interstitial alveolar infiltrates and associated with areas of virus-infected cells. There were very few CD4 $+\mathrm{T}$ cells present in the inflammatory infiltrates. The magnitude of lymphocytes in the peribronchiolar infiltrates contrasts sharply with a study of bronchial lavage samples ${ }^{19}$ in which CD3 + $\mathrm{T}$ cells were not present in BAL fluid in infected infants. The prominence of CD8 $+\mathrm{T}$ cells in the peribronchiolar and alveolar interstitial infiltrate is consistent with the correlation of CD8 + T cells with virus clearance and illness in the murine model of $\mathrm{RSV}^{29}$ and the bovine model of BRSV. ${ }^{28}$

In summary, we have presented the pathology of acute RSV infection in four infants that did not receive modern medical interventions. We have demonstrated that virus infects both the bronchial and alveolar epithelium, and that obstruction of small airways from epithelial projections, inflammatory debris, edema, or compression by lymphoid aggregates in the BALT may be an important component of disease pathogenesis. In addition, the different patterns of polymorphonuclear leukocyte distribution concentrated in and around the airway compared with the diffuse distribution of mononuclear cells throughout the airway and lung parenchyma should be considered in the development of treatment approaches for airway hyperreactivity and pneumonitis induced by RSV infection. We have shown that the monocytes, double-negative T-lymphocytes, and CD8 + T-lymphocytes are the primary cellular immune responses to human RSV infection detectable in lung parenchyma. The role of the monocytes and CD3 + double-negative $\mathrm{T}$ cells needs to be better defined, and this pattern of immune response should be considered in vaccine approaches for preventing RSV infection.

\section{Acknowledgements}

We thank Dr Robert D Collins and Dr David T Karzon for their wisdom and guidance in the retrieval and evaluation of these samples, and Monique Young for assistance with acquisition of historical references. This paper is written in tribute to the memory of Dr Ernest W Goodpasture, and with respect to other former faculty members and staff in the Department of Pathology at the Vanderbilt University School of Medicine for the care and preservation of records and tissue samples from the preintensivist era. We particularly thank Caroline (Flip) Nolen and Timothy Dolan who worked on the technical aspects of tissue retrieval and preparation. This work was supported in part by RO1 AI-33933. 


\section{References}

1 Aherne W, Bird T, Court SD, et al. Pathological changes in virus infections of the lower respiratory tract in children. J Clin Pathol 1970;23:7-18.

2 Downham MA, Gardner PS, McQuillin J, et al. Role of respiratory viruses in childhood mortality. BMJ 1975;1:235-239.

3 Ferris JA, Aherne WA, Locke WS, et al. Sudden and unexpected deaths in infants: histology and virology. BMJ 1973;2:439-442.

4 Gardner PS, Turk DC, Aherne WA, et al. Deaths associated with respiratory tract infection in childhood. BMJ 1967;4:316-320.

5 Kurlandsky LE, French G, Webb PM, et al. Fatal respiratory syncytial virus pneumonitis in a previously healthy child. Am Rev Respir Dis 1988;138:468-472.

6 Neilson KA, Yunis EJ. Demonstration of respiratory syncytial virus in an autopsy series. Pediatr Pathol 1990;10:491-502.

7 Shedden WI, Emery JL. Immunofluorescent evidence of respiratory syncytial virus infection in cases of giant cell bronchiolitis in children. J Pathol Bacteriol 1965; 89:343-347.

8 Culling CFA, Allison RT, Barr WT. Cellular Pathology Technique, 4th edn. Butterworth's: London, 1985.

9 Goodpasture EW, Auerbach SH, Swanson HS, et al. Virus pneumonia of infants secondary to epidemic infections. Am J Dis Child 1939;57:997-1011.

10 Taubenberger JK, Reid AH, Krafft AE, et al. Initial genetic characterization of the 1918 'Spanish' influenza virus [see comments]. Science 1997;275:1793-1796.

11 Philippou S, Otto P, Reinhold P, et al. Respiratory syncytial virus-induced chronic bronchiolitis in experimentally infected calves. Virchows Arch 2000;436: 617-621.

12 Fisher RG, Gruber WC, Edwards KM, et al. Twenty years of outpatient respiratory syncytial virus infection: a framework for vaccine efficacy trials. Pediatrics 1997;99:E7.

13 Zhang L, Peeples ME, Boucher RC, et al. Respiratory syncytial virus infection of human airway epithelial cells is polarized, specific to ciliated cells, and without obvious cytopathology. J Virol 2002;76:5654-5666.

14 Wright PF, Ikizler M, Carroll KN, et al. Interactions of viruses with respiratory epithelial cells. Semin Virol 1996;7:227-235.

15 Tyner JW, Kim EY, Ide K, et al. Blocking airway mucous cell metaplasia by inhibiting EGFR antiapoptosis and IL-13 transdifferentiation signals. J Clin Invest 2006;116:309-321.

16 Brock SC, Goldenring JR, Crowe Jr JE. Apical recycling systems regulate directional budding of respiratory syncytial virus from polarized epithelial cells. Proc Natl Acad Sci USA 2003;100:15143-15148.

17 Panuska JR, Cirino NM, Midulla F, et al. Productive infection of isolated human alveolar macrophages by respiratory syncytial virus. J Clin Invest 1990;86: 113-119.

18 Hornung V, Schlender J, Guenthner-Biller M, et al. Replication-dependent potent IFN-alpha induction in human plasmacytoid dendritic cells by a singlestranded RNA virus. J Immunol 2004;173:5935-5943.

19 Everard ML, Swarbrick A, Wrightham M, et al. Analysis of cells obtained by bronchial lavage of infants with respiratory syncytial virus infection. Arch Dis Child 1994;71:428-432.

20 Smith PK, Wang SZ, Dowling KD, et al. Leucocyte populations in respiratory syncytial virus-induced bronchiolitis. J Paediatr Child Health 2001;37: 146-151.

21 Hornsleth A, Loland L, Larsen LB. Cytokines and chemokines in respiratory secretion and severity of disease in infants with respiratory syncytial virus (RSV) infection. J Clin Virol 2001;21:163-170.

22 Noah TL, Ivins SS, Murphy P, et al. Chemokines and inflammation in the nasal passages of infants with respiratory syncytial virus bronchiolitis. Clin Immunol 2002;104:86-95.

23 Graham BS, Perkins MD, Wright PF, et al. Primary respiratory syncytial virus infection in mice. J Med Virol 1988;26:153-162.

24 Prince GA, Jenson AB, Hemming VG, et al. Enhancement of respiratory syncytial virus pulmonary pathology in cotton rats by prior intramuscular inoculation of formalin-inactiva ted virus (published erratum appears in J Virol 1986 July;59(1):193) J Virol 1986;57:721-728.

25 Zhang ZX, Yang L, Young KJ, et al. Identification of a previously unknown antigen-specific regulatory $\mathrm{T}$ cell and its mechanism of suppression. Nat Med 2000;6: 782-789.

26 Johnson TR, Hong S, Van Kaer L, et al. NK T cells contribute to expansion of CD8(+) T cells and amplification of antiviral immune responses to respiratory syncytial virus. J Virol 2002;76:4294-4303.

27 Openshaw PJ. Pulmonary epithelial T cells induced by viral infection express $\mathrm{T}$ cell receptors alpha/beta. Eur J Immunol 1991;21:803-806.

28 Taylor G, Thomas LH, Wyld SG, et al. Role of Tlymphocyte subsets in recovery from respiratory syncytial virus infection in calves. J Virol 1995;69: 6658-6664.

29 Graham BS, Bunton LA, Wright PF, et al. Role of T lymphocyte subsets in the pathogenesis of primary infection and rechallenge with respiratory syncytial virus in mice. J Clin Invest 1991;88:1026-1033. 